\title{
Dynamic Spike Threshold and Zero Membrane Slope Conductance Shape the Response of Subthalamic Neurons to Cortical Input
}

\author{
Michael A. Farries, ${ }^{1}$ Hitoshi Kita, ${ }^{2}$ and Charles J. Wilson ${ }^{1}$ \\ ${ }^{1}$ Department of Biology, University of Texas San Antonio, San Antonio, Texas 78249, and 2Department of Anatomy and Neurobiology, University of \\ Tennessee Health Science Center, Memphis, Tennessee 38163
}

\begin{abstract}
The subthalamic nucleus (STN) provides a second entry point for cortical input to the basal ganglia, supplementing the corticostriatal pathway. We examined the way intrinsic properties shape the response of the STN to cortical excitation, recording from rat STN in vivo and in brain slices. STN cells exhibited a near-zero slope conductance-and hence an effectively infinite membrane time constant-at subthreshold potentials. This makes STN cells exceptional temporal integrators, consistent with the common view that basal ganglia nuclei use rate coding. However, STN cells also exhibited a drop in spike threshold triggered by larger EPSPs, allowing them to fire time-locked spikes in response to coincident input. In addition to promoting coincidence detection, the threshold dynamics associated with larger EPSPs reduced the probability of firing spikes outside of a narrow window immediately after the stimulus, even on trials in which the EPSP did not directly trigger a spike. This shift in stimulus-evoked firing pattern would allow downstream structures to distinguish coincidence-triggered spikes from other spikes and thereby permit coincidence detection and rate coding to operate in parallel in the same cell. Thus, STN cells can combine two functions-integration and coincidence detection-that are normally considered mutually exclusive. This could support rapid communication between cortex and basal ganglia targets that bypasses the striatum without disrupting slower rate coding pathways.
\end{abstract}

\section{Introduction}

The subthalamic nucleus (STN) is a population of autonomously active glutamatergic neurons that innervates the output nuclei of the basal ganglia - the internal segment of the globus pallidus and the substantia nigra pars reticulata-and is reciprocally connected with the external segment of the globus pallidus (GPe). The STN is traditionally understood as a component of the indirect pathway, one of two pathways that begin with cortical projections to the striatum and, according to a widely held model, ultimately exert opposing effects on firing rates in basal ganglia output nuclei. However, the STN also receives direct cortical input (Kitai and Deniau, 1981; Canteras et al., 1990). The STN response to cortical stimulation in vivo is quite complex (Fujimoto and Kita, 1993; Kita, 1994; Magill et al., 2004) and exerts a rapid and powerful effect on other basal ganglia nuclei, including output nuclei (Ryan and Clark, 1991; Kita, 1994; Nambu et al., 2000; Kita et al., 2004). Indeed, the corticosubthalamic pathway acts on output nuclei at shorter latency and with apparently equal or greater effect than corticostriatal pathways (Kitano et al., 1998; Tachibana et al., 2008), implying a major contribution to basal ganglia function. Given the rapid and pow-

Received April 14, 2010; revised July 12, 2010; accepted Aug. 10, 2010.

This work was supported by National Institutes of Health Grant NS47085.

Correspondence should be addressed to Michael A. Farries, Department of Biology, University of Texas San Antonio, One UTSA Circle, San Antonio, TX 78249. E-mail: michael.farries@utsa.edu.

DOI:10.1523/JNEUROSCI.1909-10.2010

Copyright $\odot 2010$ the authors $\quad 0270-6474 / 10 / 3013180-12 \$ 15.00 / 0$ erful effect the corticosubthalamic pathway can exert on basal ganglia output nuclei, a full appreciation of basal ganglia processing of cortical input requires an understanding of the impact cortical input has on the activity of STN neurons.

We studied the effect of cortical input on subthalamic activity in vivo and in brain slices, allowing us to disentangle the polysynaptic contributions of basal ganglia circuitry from the interaction between direct cortical excitation and the complex intrinsic physiological properties expressed by STN cells. Basal ganglia nuclei, including the STN, are commonly assumed to use a rate code. Rate coding works best in neurons that have a long membrane time constant, allowing them to perform accurate temporal integration of synaptic inputs. We found that a balance between normal ohmic conductances and a tetrodotoxin (TTX)-sensitive negative slope conductance gives STN cells an extremely long membrane time constant, consistent with the idea that these cells act as integrators and use a rate code. However, we also found that larger EPSPs evoke a rapid and transient drop in spike threshold that allows STN cells to perform coincidence detection, a function that is normally regarded as incompatible with effective temporal integration (König et al., 1996; Kempter et al., 1998).

\section{Materials and Methods}

All experimental procedures followed National Institutes of Health guidelines and were approved by the Institutional Animal Care and Use Committees of the University of Texas San Antonio (slice recording) and the University of Tennessee Health Science Center (in vivo recording). 
Recordings in vivo. In vivo recordings were performed in four adult male Sprague Dawley rats, two with ibotenic acid lesions in the GPe. In a preparatory surgery, rats were anesthetized with an intraperitoneal injection of ketamine $(85 \mathrm{mg} / \mathrm{kg})$ and xylazine $(15 \mathrm{mg} / \mathrm{kg})$ and placed in a stereotaxic frame. A recording chamber and two stainless steel headholding tubes were fixed to the skull with dental acrylic. To implant stimulating electrodes, rats were anesthetized with isoflurane $(2.5 \%$ in $\mathrm{O}_{2}$ ) and mounted in a stereotaxic device using the stainless steel headholding tubes, and burr holes were drilled to expose lateral agranular cortex (primary motor cortex M1). The forelimb region of M1 was identified using microstimulation, and bipolar stimulating electrodes (enamel-coated $100 \mu \mathrm{m}$ stainless steel wire, $0.6 \mathrm{~mm}$ tip separation) were implanted and secured with dental acrylic. For recording, rats were anesthetized with $2.5 \%$ isoflurane, mounted in a stereotaxic device, and subsequently maintained with $1.0-1.5 \%$ isoflurane. Glass-coated elgiloy alloy microelectrodes $(1.5-2.5 \mathrm{M} \Omega$ at $1 \mathrm{kHz})$ were advanced into the STN, and signals were amplified, bandpass filtered at $0.7-2 \mathrm{kHz}$ (model 1800 AC amplifier; A-M Systems), digitized, and recorded. Spikes were detected with a window discriminator; unit isolation was confirmed by the existence of an absolute refractory period. M1 was stimulated with single pulses (300 $\mu \mathrm{s}, 20-80 \mu \mathrm{A})$ delivered every 1.4-3 s. After recording, recording sites were marked by electrolytic lesion, and locations were verified histologically. Two rats received GPe lesions 1 week before STN recording. Here, the M1-responsive region of the GPe was identified by cortical stimulation and GPe recording (following procedure used in STN recordings), and ibotenic acid ( $25 \mathrm{~mm}$ in saline, $\mathrm{pH} 7.4$ ) was injected into the targeted region at four locations $(0.1 \mu \mathrm{l} /$ injection, for a total of $0.4 \mu \mathrm{l}$ ) covering the entire M1-responsive area of the GPe. Lesion sites were verified by examination of $40 \mu \mathrm{m}$ sections, cut in the sagittal plane, and stained for neuronal-specific nuclear protein (NeuN); in each case, there was complete neuronal loss throughout the entire mediolateral extent of the GPe and adjacent parts of caudate-putamen.

Recordings in brain slices. Sprague Dawley rats of either sex, aged 16-23 $\mathrm{d}$, were deeply anesthetized and perfused transcardially with ice-cold low-sodium artificial CSF (ACSF) containing the following (in mM): 230 sucrose, $2.5 \mathrm{KCl}, 1.25 \mathrm{NaH}_{2} \mathrm{PO}_{4}, 0.5 \mathrm{CaCl}_{2}, 10 \mathrm{MgSO}_{4}, 10 \mathrm{D}$-glucose, and $26 \mathrm{NaHCO}_{3}$. Brains were rapidly removed and sectioned at $350 \mu \mathrm{m}$ with a vibrating microtome; the plane of section was rotated $\sim 15^{\circ}$ off the parasagittal plane such that more rostral regions of the slice were displaced laterally. Slices were collected into normal ACSF containing the following (in mM): $126 \mathrm{NaCl}, 2.5 \mathrm{KCl}, 1.25 \mathrm{NaH}_{2} \mathrm{PO}_{4}, 2 \mathrm{CaCl}_{2}, 2 \mathrm{MgSO}_{4}$, $10 \mathrm{D}$-glucose, and $26 \mathrm{NaHCO}_{3}$. Slices were stored at room temperature while ACSF was bubbled continuously with a $95 \% \mathrm{O}_{2} / 5 \% \mathrm{CO}_{2}$ mixture. For recording, slices were submerged and superfused continuously with oxygenated ACSF heated to $30-32^{\circ} \mathrm{C}$. Recording pipettes were fabricated with a Flaming-Brown pipette puller (model P-97; Sutter Instruments) to obtain tip diameters of 1-3 $\mu \mathrm{m}$ (electrode resistance, 2-6 M $\Omega$ ). Cells were visualized with an Olympus BX50WI microscope equipped with a $40 \times$ water-immersion objective and differential interference contrast optics. For most whole-cell recording, recording pipettes were filled with a solution containing the following (in $\mathrm{mM}$ ): $140 \mathrm{~K}$-methylsulfate, 10 HEPES, 0.2 EGTA, 7.5 NaCl, $2 \mathrm{MgATP}$, and $0.2 \mathrm{Na}_{2} \mathrm{GTP}$. To study postsynaptic currents in voltage clamp, an alternative cesium-based solution was used instead, containing the following (in $\mathrm{mm}$ ): $135 \mathrm{CsCl}, 5$ QX-314 (lidocaine $N$-ethyl bromide), 10 HEPES, 5 EGTA, $2 \mathrm{MgCl}_{2}, 2$ MgATP, and $0.2 \mathrm{Na}_{2} \mathrm{GTP}$. ATP and GTP were often omitted from pipette solutions used in perforated-patch and cell-attached recordings. For perforated-patch recordings, a stock solution of gramicidin-D $(10 \mathrm{mg} / \mathrm{ml}$ in DMSO) was prepared daily; $1-5 \mu l$ of this stock was added to pipette solution (final concentration of $20-100 \mu \mathrm{g} / \mathrm{ml}$ ) and sonicated no more than $1 \mathrm{~h}$ before use. Electrodes were front-filled with gramicidin-free solution and backfilled with solution containing gramicidin. Data were recorded with a MultiClamp 700B amplifier (Molecular Devices), lowpass filtered at $4-10 \mathrm{kHz}$, and acquired at $10-50 \mathrm{kHz}$ with an ITC-18 analog-to-digital converter (InstruTECH Corporation). Synaptic responses were evoked with bipolar tungsten microelectrodes (tip spacing, $250-500 \mu \mathrm{m}$ ) placed in the internal capsule, typically $>1 \mathrm{~mm}$ rostral and dorsal to the STN; stimuli were $100 \mu$ s pulses, $0.1-8 \mathrm{~mA}$. Drugs used in this study included picrotoxin, TTX, 6,7-dinitroquinoxaline-2,3(1 H,4H)-dione
(DNQX), DL-2-amino-5-phosphonovaleric acid (APV), and CGP-55845 [(2S)-3-[(15)-1-(3,4-dichlorophenyl)ethyl] amino-2-hydroxypropyl) (phenylmethyl)phosphinic acid]. All were obtained from Sigma, save CGP55845 (Tocris Bioscience).

Voltage-clamp recording in the whole-cell configuration was used to study the postsynaptic currents evoked by stimulation of the internal capsule and to measure slope conductance in a sample of STN cells. Series resistance was measured on every trial; only data obtained with series resistance $<20 \mathrm{M} \Omega$ were retained for analysis. Pipette capacitance was compensated, and series resistance voltage error was corrected offline; voltage was also corrected for the liquid junction potential $(7 \mathrm{mV}$ for $\mathrm{K}$-methylsulfate internal, $6 \mathrm{mV}$ for $\mathrm{CsCl}$ internal) and postrecording voltage offset (typically $\pm 0-5 \mathrm{mV}$ ). Voltage ramps from $-64 \pm 3$ to $-31 \pm 2 \mathrm{mV}$ (after voltage corrections) at $0.1 \mathrm{~V} / \mathrm{s}$ were used to measure current-voltage $(I-V)$ curves. For current-clamp recording, series resistance was monitored frequently, and error was corrected using the bridge circuit of the amplifier; pipette capacitance was also compensated. If necessary, remaining bridge error was corrected offline ( $<10 \mathrm{M} \Omega$ offline compensation). Voltages were corrected for postrecording offset and, for whole-cell recordings, the liquid junction potential.

Data analysis. Data are presented as mean $\pm \mathrm{SD}$ unless otherwise noted. Most data analysis was performed using custom algorithms implemented in Mathematica (Wolfram Research). $t$ tests were performed in Prism (GraphPad Software). Unimodality of spike latency distributions was assessed using Hartigan's dip test (Hartigan and Hartigan, 1985) implemented in R, a freely available statistical computing environment (http://www.r-project.org). Hartigan's dip test examines the null hypothesis that a dataset (of latency to first poststimulus spike, in our case) was drawn from a unimodal underlying distribution. If the null hypothesis was rejected at a significance criterion of $p<0.05$, we concluded that the underlying distribution was multimodal (our latency data generally appeared bimodal when the unimodal hypothesis was rejected).

Postsynaptic currents were studied using whole-cell recording with a cesium-based internal solution (see above). Decay time constants of postsynaptic currents were measured by fitting a single exponential to the decay phase of stimulation-evoked currents, with AMPA-type receptor (AMPAR) currents recorded at hyperpolarized potentials $(-85$ to -65 $\mathrm{mV}$ ) and NMDA-type receptor (NMDAR) currents recorded at depolarized potentials $(+20$ to $+40 \mathrm{mV}$ ). The NMDA/AMPA ratio is defined as the ratio of peak conductances for the NMDAR- and AMPAR-mediated components of the synaptic response. The AMPAR-mediated conductance was estimated from currents recorded at hyperpolarized potentials (less than $-65 \mathrm{mV}$ ). The NMDAR-mediated conductance was estimated from currents recorded at depolarized potentials (more than $+20 \mathrm{mV}$ ) in the presence of $10 \mu \mathrm{M}$ DNQX $(n=3)$ or from the difference between currents recorded in control conditions and currents recorded in $50 \mu \mathrm{M}$ APV (the APV-sensitive component, $n=4$, again measured at holding potentials more than $+20 \mathrm{mV}$ ).

Spike thresholds reported in this paper were defined as the voltage at which the second derivative of the membrane potential trajectory approaching a spike crossed a user-selected threshold of $50 \mathrm{mV} / \mathrm{ms}^{2}$. Given the importance of threshold measurements in this work, we also measured spike threshold in all our data with second-derivative thresholds of 20 and $100 \mathrm{mV} / \mathrm{ms}^{2}$. We also used an alternative definition of spike threshold, namely, the voltage at which the first derivative of the membrane potential trajectory crossed user-selected thresholds of 10 or 20 $\mathrm{V} / \mathrm{s}$, again applied to all our data. Although the absolute spike threshold measured using these differing techniques and derivative thresholds varied, the relative spike thresholds (i.e., of stimulus-evoked spikes compared with spontaneous spikes) was virtually unchanged. That is, changing the measurement method changed the measured spike threshold, but it changed the measured threshold of all spikes uniformly and thus had no impact on our conclusions. To measure the peak threshold drop after stimulation, poststimulus spikes were divided into $0.5 \mathrm{~ms}$ bins; the bin with the lowest average threshold was identified, and the peak threshold drop was defined as the average threshold in that bin (relative to spontaneous spike threshold). In a few cases, bins of 1 or $2 \mathrm{~ms}$ were used to ensure that the peak bin contained at least three spikes. The decay 
phase of the threshold drop (from the peak drop to $50 \mathrm{~ms}$ after the stimulus) was fit with a single exponential to quantify its duration (decay time constant). The peak threshold rise that often followed threshold drops was analyzed in the same way, save that spikes were divided into $25 \mathrm{~ms}$ bins.

Because EPSPs in STN cells usually do not decay, defining the EPSP amplitude is not straightforward. For each cell, we examined the average EPSP after stimulation on trials in which the latency to the first poststimulus spike was $>20 \mathrm{~ms}$. The average EPSP trajectory, along with its first and second derivatives, was used to identify the average time of EPSP onset (typically 1-4 ms after the stimulus). The peak time of the average EPSP was identified as the time when the EPSP slope stopped decaying after its peak, approximately when the second derivative of the voltage trajectory crossed zero. This peak time was typically $3-10 \mathrm{~ms}$ after EPSP onset. EPSP amplitude is defined as difference between the potential at peak time and the potential at onset time. EPSP decay is defined as the minimum potential occurring in the interval from $1 \mathrm{~ms}$ after EPSP peak time to the time of the next spike, minus the EPSP onset potential. The EPSP fractional decay is the EPSP decay divided by the EPSP amplitude. A fractional decay of 0.5 , for example, means that the EPSP decayed halfway back to the onset potential before resuming its depolarizing trajectory to the next spike. A fractional decay $>1$ corresponds to depolarization occurring in the $1 \mathrm{~ms}$ between the EPSP peak and the beginning of the decay measurement window, in cases when the potential in the decay window is at all points more depolarized than the potential at the EPSP peak. The peak EPSP slope was the maximum $d V / d t$ occurring between EPSP onset and EPSP peak.

Modeling STN cells and spike threshold. We developed a very simple model of the STN cell and its response to excitatory synaptic input to test our intuitions about spike latency and the effects of spike threshold. This model is based on the observations that (1) in the absence of synaptic input, STN cells are spontaneously active with a stereotyped interspike voltage trajectory (see Fig. 3A), and (2) EPSPs in STN cells cause a rapid depolarization followed (approximately) by a resumption of the prestimulus rate of depolarization. To construct the model, we averaged the voltage trajectories of an STN cell over several interspike intervals (ISIs) of nearly equal duration (195-200 ms) and took its derivative to obtain the drive attributable to intrinsic mechanisms (Fig. $1 A$, top left). To obtain synaptic drive, we took the average EPSP from an STN cell (over traces in which the spike latency was $>20 \mathrm{~ms}$ ), computed its derivative, and subtracted the baseline $d V / d t$ so that it starts from a depolarization rate of zero (Fig. $1 \mathrm{~A}$, middle left). EPSPs of any size are obtained by scaling this synaptic drive waveform up or down. To simulate the arrival of an EPSP, the synaptic drive is added to the intrinsic drive at any time in the ISI we desire; this gives the $d V / d t$ for the simulated ISI (Fig. $1 \mathrm{~A}$, bottom left). To obtain the simulated voltage trajectory, we integrate this waveform, starting at an initial membrane potential of $-55 \mathrm{mV}$ (Fig. $1 \mathrm{~A}$, right). A spike is said to occur when the voltage trajectory crosses a threshold, which may be static (Fig. $1 A$, right) (see Fig. $4 D$ ), follow a preset time course relative to the EPSP (see Fig. $8 A$ ) or obey its own dynamics (Fig. $1 B$ ) (see Fig. $8 B-D$ ). In simulations featuring threshold changes that follow a preset time course relative to the EPSP, the threshold is modeled as a linear drop followed by an exponential decay back to the baseline threshold (see Fig. $8 \mathrm{~A}$, left, red trace), using parameters consistent with experimental data $(10-90 \%$ rise time of $2 \mathrm{~ms}$, peaking $2 \mathrm{~ms}$ after peak EPSP slope, and decaying with a $5 \mathrm{~ms}$ time constant unless otherwise noted).
To model threshold changes during arbitrary input patterns, we treated spike threshold as a dynamical variable. Spike threshold $(\theta)$ obeys a linear first-order differential equation:

$$
\frac{d \theta}{d t}=\frac{\theta_{\mathrm{SS}}-\theta}{\tau_{\theta}},
$$

where $\tau_{\theta}$ is a decay time constant, and $\theta_{\mathrm{SS}}$ is the steady-state spike threshold. $\theta_{\mathrm{SS}}$ is a linear function of membrane potential: $\theta_{\mathrm{SS}}(V)=$ $\theta_{\text {base }}-k\left(\theta_{\text {base }}-V\right)$, where $\theta_{\text {base }}$ is a baseline threshold that is approximately_but not exactly-equivalent to the spontaneous spike threshold (spontaneous threshold is found numerically by running the model in the absence of synaptic input). Realistically, $\theta_{\mathrm{SS}}$ must saturate as hyperpolarization increases and is probably better represented as a sigmoidal rather than linear function of voltage. However, because voltage never drops below $-63 \mathrm{mV}$ in our model (we do not include inhibition), a linear function sufficed. At the beginning of each ISI, $\theta$ is initialized to the spontaneous threshold, which is $\sim 6.5 \mathrm{mV}$ more depolarized than the steady-state threshold at the initial membrane potential $(-55 \mathrm{mV})$, given our parameter choices. From this initial state, $\theta$ decays rapidly to a value near the steady-state threshold (Fig. $1 B$, right); this models the reduced capacity for threshold drops we observed for spikes evoked $<10 \mathrm{~ms}$ after the preceding spike. This simple model captures most dynamic threshold phenomena we observed with just three free parameters: $\tau_{\theta}, \theta_{\text {base }}$ and $k$ (with values of $6 \mathrm{~ms},-35 \mathrm{mV}$, and 0.4 , respectively).

\section{Results}

\section{Response of STN cells to cortical stimulation in vivo}

We recorded seven neurons in vivo in the forelimb region of the STN in intact animals. These cells were spontaneously active, with baseline firing rates of $9.5 \pm 3.8 \mathrm{~Hz}$ (range, 4.7-16.3 Hz). This activity was highly irregular, exhibiting ISI distributions with coefficients of variation $(\mathrm{CV})$ of $1.28 \pm 0.47$ (range, $0.86-$ 2.27). The response of these cells to stimulation of the forelimb region of primary motor cortex was complex and comprised several distinct phases (Fig. $2 \mathrm{~A}$ ). The earliest phases consisted of a 
A

intact GPe population data $(\mathrm{n}=7)$
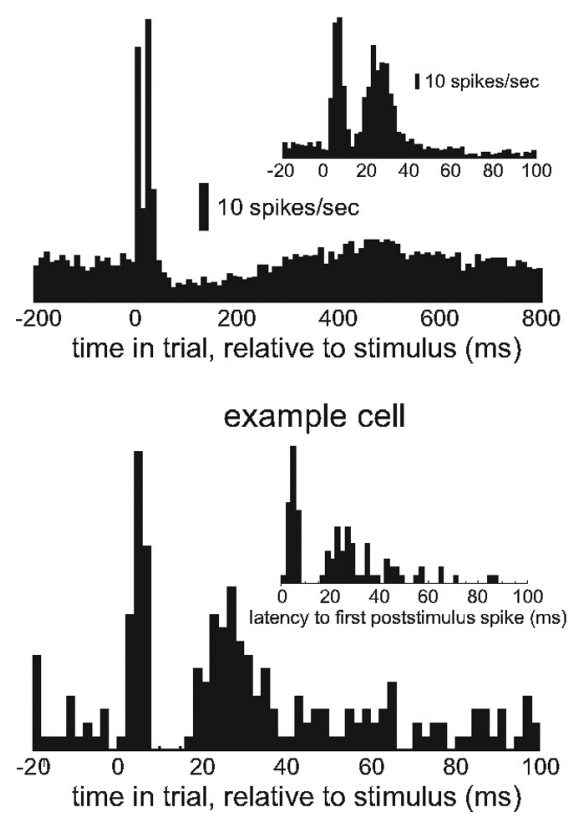

B
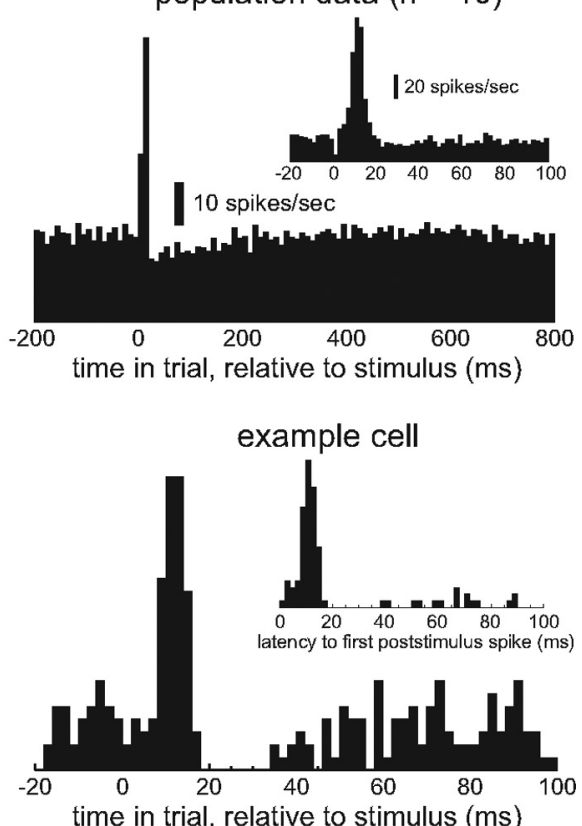

Figure 2. Response of STN cells to cortical stimulation in vivo. A, Top, Combined PSTH from seven STN cells ( 99 trials per cell) during cortical stimulation delivered at time $0,10 \mathrm{~ms}$ bins. Top inset shows detail of the first $100 \mathrm{~ms}$ of the response with $2 \mathrm{~ms}$ bins. Bottom, PSTH showing early response (first $100 \mathrm{~ms}, 2 \mathrm{~ms}$ bins) of an example cell. Bottom inset, Latency to first poststimulus spike, $2 \mathrm{~ms}$ bins. $\boldsymbol{B}$, As in $\boldsymbol{A}$ but from rats with lesioned GPe. Apparent single-bin reduction in firing visible at time of stimulus in the top inset is attributable to exclusion of stimulus artifact.

sharp and brief increase in firing probability, beginning 3-5 ms after stimulation and lasting $4-8 \mathrm{~ms}$, followed by a transient reduction in firing probability (5-15 ms duration) and then a late increase in firing probability lasting $\sim 20 \mathrm{~ms}$ (Fig. $2 \mathrm{~A}$, top inset and bottom). These early phases were followed by long-lasting reduction in firing and mild rebound (Fig. $2 A$, top). This pattern of response matches what has been reported before for the STN response to cortical stimulation in rats (Ryan and Clark, 1991; Fujimoto and Kita, 1993; Magill et al., 2004) and monkeys (Nambu et al., 2000). The early increase in firing probability is almost certainly attributable to monosynaptic excitation from the cortex, whereas the brief reduction in firing probability that follows is generally attributed to polysynaptic inhibition from the GPe.

We recorded 10 neurons from the forelimb region of the STN in rats that had received a large excitotoxic lesion of the GPe, encompassing the entire mediolateral extent of the GPe and adjacent parts of the caudate-putamen. STN cells in these rats fired at a higher rate $(20.7 \pm 5.2 \mathrm{~Hz}$; range, $9.8-30.4 \mathrm{~Hz})$ and with greater regularity $(\mathrm{CV}, 0.53 \pm 0.10$; range, $0.40-0.74)$ than unlesioned rats (both differences statistically significant, unpaired $t$ tests, $p<0.001)$. Cortical stimulation evoked an early and brief increase in firing probability, as in intact animals, but the brief reduction in firing probability (and later increase in firing) could not be seen in the peristimulus time histogram (PSTH) of all cells combined (Fig. $2 B$, top), consistent with the hypothesis that inhibition from the GPe is responsible for the brief decrease in firing. Nevertheless, some cells continued to exhibit a reduction in firing probability $15-40 \mathrm{~ms}$ after cortical stimulation (Fig. 2 B, bottom). This could be attributed to spikes fired at short latency after stimulation, i.e., given that the cell fires 5-15 ms after the stimulus on most trials, the cell will usually be undergoing an

afterhyperpolarization or experiencing spike refractoriness $15-40 \mathrm{~ms}$ after the stimulus, and thus will be much less likely to fire a second spike during that time. However, the distribution of times to the first poststimulus spike (Fig. $2 B$, bottom inset) suggests that this is not the full story; a gap in this distribution $15-40 \mathrm{~ms}$ after the stimulus cannot be explained by the effect of a preceding poststimulus spike in the recorded cell. In animals with an intact GPe, such a gap (Fig. $2 \mathrm{~A}$, bottom inset) is easily explained by inhibition from the GPe, but in GPe-lesioned animals the explanation is not so clear. To identify the processes that might shape the response to cortical stimulation even in the absence of inhibition from the GPe, we studied the responses of STN cells to stimulation of the corticosubthalamic pathway in brain slices.

\section{Response of STN cells to internal capsule stimulation in brain slices} We recorded a total of 112 STN cells in rat brain slices, using cell-attached, wholecell, and perforated-patch recording configurations. To assess the spiking response of STN cells to cortical excitation, we recorded 90 STN cells in current-clamp mode or in the cell-attached configuration in the presence of GABA antagonists (the $\mathrm{GABA}_{\mathrm{A}}$ channel blocker picrotoxin at $150 \mu \mathrm{M}, n=10$; or $150 \mu \mathrm{M}$ picrotoxin with the $\mathrm{GABA}_{\mathrm{B}}$ receptor antagonist CGP-55845 at $1-2 \mu \mathrm{M}, n=80$ ). These cells were spontaneously active in brain slices (10.6 \pm 6.0 $\mathrm{Hz}$; range, $1.8-26.5 \mathrm{~Hz})$ and fired very regularly $(\mathrm{CV}, 0.14 \pm 0.08$; range, $0.06-0.51$ ); an example trace is shown in Figure $3 A$. The average PSTH of the response to stimulation of the internal capsule in brain slices resembled the average PSTH of the response to cortical stimulation in vivo after GPe lesions: a single sharp and brief increase in firing probability (Fig. 3B).

Although the PSTH of all cells combined (Fig. $3 B$ ) showed only an increase in firing probability followed by a return to baseline, the PSTHs of individual cells often displayed an early increase in firing probability followed by a brief reduction in firing probability beginning $10-30 \mathrm{~ms}$ after the stimulus and lasting 5-25 ms (Fig. 3C). A strong stimulus can evoke two or more spikes at short latency, and the afterhyperpolarization or refractory period associated with the first spike could cause a transient reduction in firing probability. However, the distribution of time to first poststimulus spike (Fig. 3C, insets) is bimodal in many cells, showing an early peak and a long tail separated by a 5-25 ms gap during which the first poststimulus spike is unlikely to occur; this pattern cannot be explained by a reduction in firing probability triggered by a preceding spike fired at short latency. Because the timing and duration of the gap in the spike latency distribution is variable and because some cells do not exhibit this gap, it is not evident in the latency distribution for all cells combined (data not shown). However, the latency distribution of individual cells can be tested for unimodality, and this analysis shows that the latency distribution of $71 \%$ of cells is bimodal or multimodal (Hartigan's dip test, $p<0.05$ significance criterion). Both in vivo and in brain slices, cortically driven exci- 
tation in STN cells is often followed by an inhibition-like phenomenon even when GABAergic synaptic inhibition is compromised by lesion (in vivo) or blocked pharmacologically (slices).

To determine whether the reduction in firing probability after stimulation in the internal capsule might be explained by non-GABAergic inhibition or unusual properties of glutamatergic synaptic transmission in STN cells, we examined postsynaptic currents evoked by internal capsule stimulation under voltage clamp in the whole-cell configuration in the presence of GABA antagonists $(150 \mu \mathrm{M}$ picrotoxin and $1 \mu \mathrm{M}$ CGP-55845). At hyperpolarized holding potentials $(-65 \mathrm{mV}$ or below), stimulation evoked an inward current (Fig. 3D, left) that decayed with a time constant of $2.49 \pm 0.75 \mathrm{~ms}(n=11$; range, 1.65-3.64 ms) and was blocked by the AMPA-type glutamate receptor antagonist DNQX $(10 \mu \mathrm{M} ; n=5)$. At more depolarized potentials, a much slower synaptic current was visible that was not blocked by DNQX (Fig. 3D, top right). This component of the response decayed with a time constant of $90.1 \pm 34.0 \mathrm{~ms}$ ( $n=8$; range, 49.5-152.4 ms) and was abolished by the NMDA-type glutamate receptor antagonist APV $(50 \mu \mathrm{M} ; n=7)$. The APV-sensitive component of the EPSC exhibited a nonlinear voltage dependence characteristic of NMDA-type receptors (Fig. $3 E$ ). We saw no sign of an inhibitory component to the postsynaptic current nor anything inconsistent with a response mediated entirely by AMPAand NMDA-type glutamate receptors. The NMDA/AMPA ratio was $1.02 \pm 0.32$ $(n=7$; range, $0.63-1.33)$.

\section{STN cells have a near-zero slope} conductance over most of their ISI

The gap in the distribution of latency to first poststimulus spike that gives the appearance of inhibition might arise from an interaction between synaptic excitation and the intrinsic physiological properties of STN cells. To address this possibility, we examined the membrane potential trajectory after stimulus-evoked EPSPs (32 cells recorded via whole cell or perforated patch). When an EPSP arrives, one of two things will happen: either the EPSP itself drives the membrane potential above threshold and triggers a spike at short latency, or it fails to trigger a spike directly but may still influence the time of the next spike. The explanation for the gap in the latency distribution may lie in what happens when EPSPs do not immediately trigger spikes, and examination of subthreshold EPSPs will at least shape our expectations for the latency distribution. One striking fact that emerges from examination of such EPSPs is that they do not decay; after the rapid synaptically driven depolarization is complete, the membrane potential continues to depolarize at rates comparable with that of unperturbed ISIs rather than decaying back to a prestimulus equilibrium (Fig.

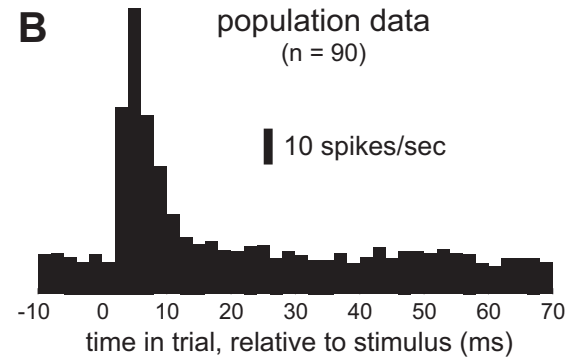

example cells
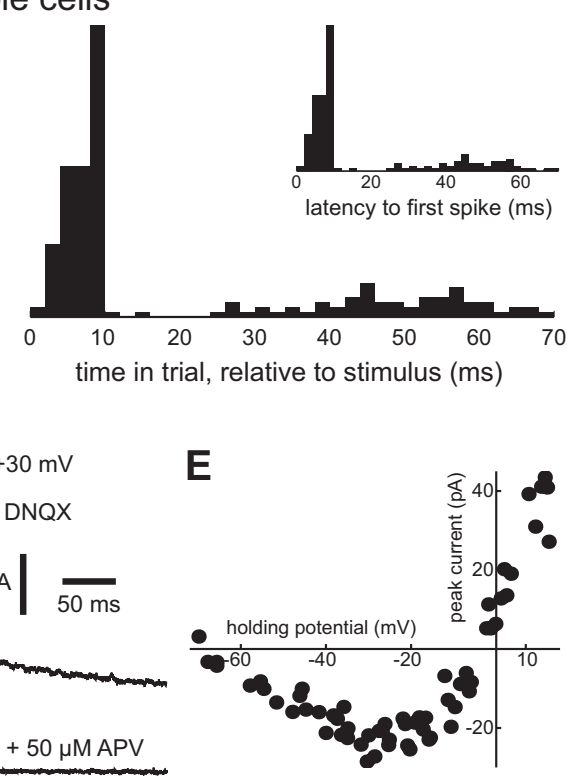

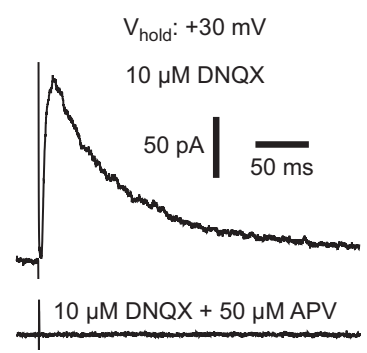

Figure 3. Response of STN cells to internal capsule stimulation in brain slices. $\boldsymbol{A}$, Example trace from an STN cell recorded in a marked by the arrowhead (a small stimulus artifact is also visible), evoking an EPSP in the recorded cell at a latency of $\sim 3 \mathrm{~ms}$. $\boldsymbol{B}$, Combined PSTH from 90 STN cells ( 50 trials per cell) recorded in brain slices during internal capsule stimulation delivered at time 0 lutamatergic EPSCs evoked by stimulation of the internal capsule. Left, Rapidly decaying inward synaptic current recorded when Vas held at $-65 \mathrm{mV}$, average of 10 traces. Right, Slowly decaying outward synaptic current when this cell was held at +30 of the NMDAR antagonist APV (bottom, average of 10 traces; both traces shown on the same scale). $\boldsymbol{F}$ ( current-voltage curve for NMDAR-mediated EPSCs. Data from seven cells are shown; to control for variation in EPSC size from cell to cell, the peak current is normalized by the individual cell NMDAR conductance relative to the NMDAR conductance averaged across cells, measured at more than $+20 \mathrm{mV}$. Each point represents data from one cell at one holding potential (average of 5-10 traces); holding potential is corrected for series resistance error, postrecording offset, and liquid junction potential.

4A). We quantify EPSP decay by measuring the fraction of the total EPSP amplitude remaining after the potential has dropped to the minimum voltage occurring from $1 \mathrm{~ms}$ after the end of the EPSP to the time of the next spike. An EPSP with a fractional decay of 0 decayed back to the prestimulus potential, a fractional decay of 1 means the EPSP did not decay at all, and a fractional decay $>1$ corresponds to uninterrupted depolarization from the end of the EPSP. By this measure, EPSPs decay very little if at all in STN cells (within-cell mean fractional decay, $1.02 \pm$ 0.07 ; range, $0.85-1.17$ )

The behavior of the membrane potential during an EPSP can be understood with the aid of instantaneous $I-V$ curves. An instantaneous $I-V$ curve represents the response of the cell to fast perturbations such as EPSPs; channel gating variables with relatively slow kinetics (e.g., $\mathrm{Na}^{+}$channel inactivation) are assumed to remain constant on the timescale of the perturbation, whereas fast voltage-dependent variables (e.g., $\mathrm{Na}^{+}$channel activation) 

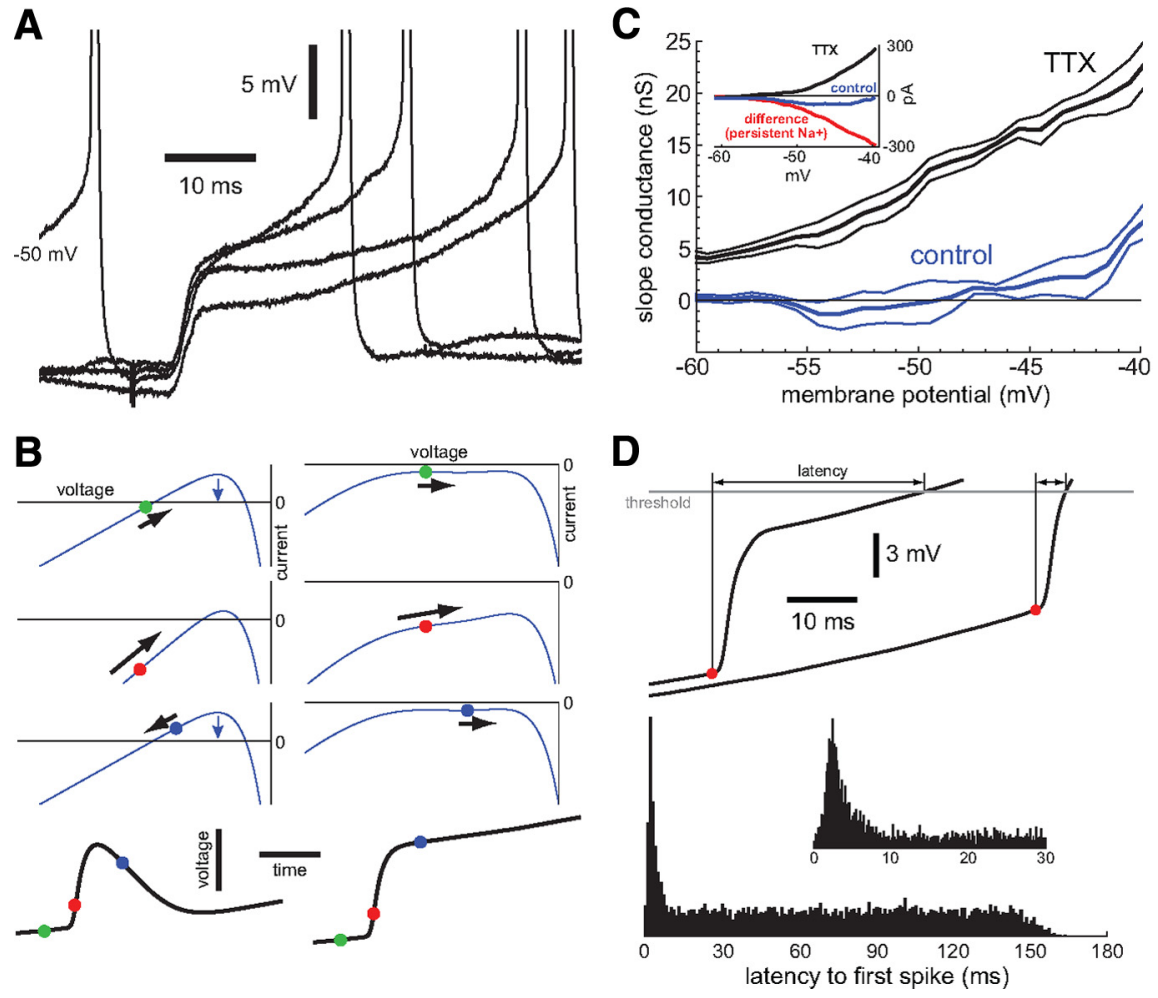

Figure 4. STN cells exhibit a near-zero slope conductance in the subthreshold range that prevents EPSPs from decaying. $\boldsymbol{A}$, EPSPs recorded in an STN cell, evoked by stimulation in the internal capsule. $\boldsymbol{B}$, Schematic instantaneous $I-V$ curves and resulting voltage trajectories (bottom), illustrating EPSP generation. Within each column, the three $I-V$ curves represent different time points during the EPSP (corresponding to the green, red, and blue circles on the EPSP voltage trajectories). Black arrows represent the direction and rapidity of voltage changes. Left column, Sequence of events during an EPSP in a cell with a normal positive-slope membrane conductance. Spontaneous activity is driven by slow membrane changes that cause a gradual drop in the $I-V$ curve over time, represented by the small blue arrows. Right column, Corresponding sequence of events when the cell has a near-zero slope conductance. C, Slope conductance measured in six STN cells, plotted as a function of membrane potential. Thick lines show mean slope conductance across all cells, and thin lines show the SEM. Measurements shown in blue are taken from control conditions, and black lines show the same measurements taken in the presence of $1 \mu \mathrm{m}$ TTX. Inset shows $1-V$ curves from an example cell, measured with voltage ramps (each an average of 10 traces). The control/-V curve (blue), with a slope close to zero, is derived from a combination of TTX-insensitive positive-slope conductances (black) and a $\Pi$ TX-sensitive negative slope conductance (red, difference current of control - TTX, presumably persistent $\mathrm{Na}^{+}{ }^{+}$current). D, A simpleSTN model and its predicted spike latency distribution. Top, Basic operation of the model. Synaptic input triggers brief increases in depolarization rate, after which the membrane potential resumes its former course. EPSPs will either trigger a spike immediately (bottom left trajectory) or advance the timing of that spike without triggering it directly (top right trajectory). Red circles mark the onset of the simulated EPSP. Bottom, Distribution of latency to first poststimulus spike predicted by this model ( 1000 trials, $1 \mathrm{~ms}$ bins), showing an early peak generated by EPSPs that directly trigger spikes and a long tail associated with those that do not. Inset shows detail of early peak ( $0.2 \mathrm{~ms}$ bins).

are treated as direct functions of voltage and are incorporated into the curve. The dynamics of slower conductances are represented by changes in the shape of the instantaneous $I-V$ curve over time. Hypothetical instantaneous $I-V$ curves are shown in Figure $4 B$ (left column), consisting of a positive slope region with a stable equilibrium in which current is zero and, at more depolarized potentials, a negative slope region that reflects $\mathrm{Na}^{+}$channel activation. Slow changes underlying the pacemaking activity of the cell are represented by a gradual drop of the entire $I-V$ curve, which causes a slow depolarization as the equilibrium potential rises (Fig. $4 B$, top left); once the peak of the curve drops below the zero current line, there is no stable equilibrium and the cell is committed to spiking. Activation of an excitatory synaptic conductance increases the slope of the $I-V$ curve and pulls it down (attributable to the depolarized reversal potential of the newly added synaptic conductance), generating a large inward current at a potential that was once near equilibrium and causing rapid depolarization (Fig. $4 B$, middle left $I-V$ curve). This leaves the cell above equilibrium after the synaptic con- ductance disappears, and thus the potential must decay back to the slowly rising equilibrium voltage (Fig. $4 B$, bottom left $I-V$ curve).

That scenario evidently does not apply to STN cells, given that their EPSPs do not decay. The reason may lie with the expression by STN of the persistent $\mathrm{Na}^{+}$current, which supplies the depolarizing drive that makes STN cells autonomously active pacemakers (Bevan and Wilson, 1999; Do and Bean, 2003). Because the persistent $\mathrm{Na}^{+}$current is a depolarization-activated depolarizing current that is active at subthreshold potentials, it effectively reduces the membrane conductance. If the persistent $\mathrm{Na}^{+}$current is powerful enough, its negative slope conductance could balance the positive slope conductances of the STN cell to produce an instantaneous $I-V$ curve with a broad zero-slope plateau (Fig. $4 B$, right). In this scenario, there is no equilibrium potential, and the membrane slowly depolarizes because the $I-V$ curve plateau lies just below the zero current line, giving a small inward current (Fig. $4 B$, top right). Activation of an excitatory synaptic conductance increases the rate of depolarization as before (Fig. $4 B$, middle $I-V$ curves), but when that conductance is removed, the cell will still be below the zero current line (still depolarizing) no matter how large the EPSP (Fig. $4 B$, bottom right $I-V$ curve). To determine whether the persistent $\mathrm{Na}^{+}$current could have this effect in the STN, we used slow $(0.1 \mathrm{~V} / \mathrm{s})$ voltage ramps delivered in voltage clamp to measure the slope conductance of STN cells as a function of voltage, where the slope conductance is the derivative of the ramp-derived $I-V$ curve with respect to voltage. We found that STN cells maintain a near-zero slope conductance over most of the subthreshold ISI voltage range (typically -60 to $-40 \mathrm{mV}$ ) (Fig. $4 C$, blue trace) and that the slope conductance becomes large and positive $(>5 \mathrm{nS})$ when $\mathrm{Na}^{+}$channels are blocked (Fig. $4 C$, black trace). The zero slope conductance of the STN cells gives them an effectively infinite membrane time constant and allows them to integrate synaptic inputs without leakage over time through the somatic membrane conductance.

It is unlikely that a zero slope conductance could explain the bimodal distribution of latency to first spike we observed in many STN cells. Furthermore, none of our observations of subthreshold EPSPs or studies of subthreshold membrane properties suggest an explanation for this phenomenon. Nevertheless, we can ask what kind of latency distribution we should expect using a simple model based on our observations. In this model, we assume that an EPSP triggers a transient increase in depolarization rate $(d V / d t)$ that is added to the intrinsic depolarization rate of the cell, which is otherwise unperturbed by the EPSP (model shown in Fig. 1A). EPSPs vary in amplitude and arrive at random phases during the ISI; latency is the time from EPSP onset to the 
time when the voltage crosses a fixed threshold (Fig. 4D, top shows example traces generated by this model). The resulting latency distribution (Fig. $4 D$, bottom) is unimodal but skewed; the main peak corresponds to spikes directly triggered by the EPSP, whereas the tail contains spikes triggered sometime after the EPSP ended.

\section{EPSPs in STN cells are accompanied by} a brief drop in spike threshold

This very simple model makes several assumptions that, if untrue, could contribute to its erroneous predictions about the spike latency distribution of stimulated STN cells. Correcting many of these assumptions would entail biophysically realistic modeling that abandons the lucidity and tractability of the simple model. However, the assumption that the spike threshold is fixed regardless of how the spike was triggered (spontaneously or by EPSP) can be relaxed without abandoning this modeling approach, and this is a factor that is easily measured and is likely to influence spike latency. When we compared spontaneous spikes to the first spike occurring after stimulation, we found that poststimulus spikes were often triggered at a lower threshold than spontaneous spikes, albeit with a considerable amount of variation from trial to trial (Fig. $5 A$ ). Most of the variation in the threshold of poststimulus spikes is explained by the time of the spike relative to the stimulus: when the threshold of a spike is plotted as a function of when the spike occurred, we discover that spike threshold drops sharply after stimulation and then rapidly rises again (Fig. $5 B$, left). The threshold rise often overshoots the spontaneous spike threshold and ends with a small elevation in spike threshold that decays over hundreds of milliseconds (Fig. 5B, right).

The peak EPSP-evoked threshold drop varied widely from cell to cell, ranging from -0.55 to $-6.60 \mathrm{mV}$ relative to the spontaneous threshold $(-2.68 \pm 1.62 \mathrm{mV}$ ) (Fig. 6A, left). In most cases, the threshold drop decayed rapidly (decay time constant, $8.6 \pm$ $8.4 \mathrm{~ms}$; range, $1.2-47.7 \mathrm{~ms}$ ) (Fig. $6 A$, left). The peak rise in threshold that generally followed the drop was smaller $(0.64 \pm$ $0.47 \mathrm{mV}$; range, $0-1.46 \mathrm{mV}$ ) but decayed more slowly (decay time constant, $126 \pm 102 \mathrm{~ms}$; range, 15-393 ms, excluding two cells that showed no detectable rise in threshold) (Fig. 6A, right). Stronger EPSPs triggered larger threshold drops, whether EPSP strength was measured by the average potential change (EPSP amplitude) (Fig. $6 B$, left) or peak depolarization rate (peak EPSP slope) (Fig. $6 B$, middle; these two measures are highly correlated, see $B$, right). To evaluate the time course of the threshold drop relative to that of the EPSP, we compared the threshold drop with the average EPSP of traces with spike latencies $>20 \mathrm{~ms}$, so that the EPSP trajectory was not contaminated with spikes. The threshold drop began almost immediately after EPSP onset and achieved a minimum $2.08 \pm 1.16 \mathrm{~ms}$ after the EPSP slope peaked (range, 0.29-6.67 ms) (Fig. $6 C$, right) but generally before the end of the EPSP-driven depolarization (Fig. 6C).

\section{Synaptically evoked changes in spike threshold can be} explained by the somatic membrane potential trajectory To determine whether the threshold changes associated with EPSPs might be explained by the somatic membrane potential trajectory, we examined the effect of current pulses on spike threshold. To avoid any error in threshold measurements introduced by imperfect bridge balance, we initially used very brief current pulses $(0.5-2 \mathrm{~ms}$, $50-800 \mathrm{pA}$ ) and examined only spikes initiated after current pulses ended (example shown in Fig. 7A). Spikes fired within $10 \mathrm{~ms}$ of current pulse offset were consistently triggered at a lower threshold $(-2.80 \pm 2.03 \mathrm{mV}$ peak threshold drop relative to spontaneous spike threshold; range, -0.34 to $-6.90 \mathrm{mV} ; n=8$ cells). Curiously, the distribution of spike latencies after brief current pulses was always bimodal with a $10-30 \mathrm{~ms}$ gap between short-latency $(<5 \mathrm{~ms})$ and longer-latency $(>15 \mathrm{~ms}$ ) spikes (example shown in Fig. $7 A$, right, unimodal null hypothesis rejected in all cases by Hartigan's dip test). A rise in spike threshold sometimes followed the threshold drop $(0.53 \pm 0.45 \mathrm{mV}$; range, $0.21-1.47 \mathrm{mV}$; five of eight cells $)$. Once we established the effect of brief current pulses on spike threshold, we examined the effect of longer current pulses (25-100 ms, 10-200 pA) (example shown in Fig. $7 B$ ). Longer pulses also elicited robust changes in spike threshold $(-2.42 \pm 1.13 \mathrm{mV}$ peak threshold drop; range, -0.75 to $-4.97 \mathrm{mV} ; n=9$ cells) that varied systematically with spike latency relative to pulse onset (Fig. $7 B$, middle and right). The subsequent threshold rise was much larger and more robust than that elicited by EPSPs or brief current pulses $(2.65 \pm 1.52 \mathrm{mV}$; range, $0.79-6.81 \mathrm{mV} ; n=9$ cells).

The effect of EPSP size on the magnitude of the threshold drop for evoked spikes (Fig. 6B) could be attributable to the rate of depolarization or the magnitude of depolarization. To examine the relationship between these factors and spike threshold, we 
A

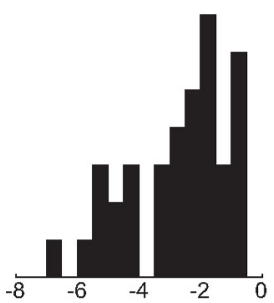
peak threshold drop $(\mathrm{mV})$ decay time constant $(\mathrm{ms})$
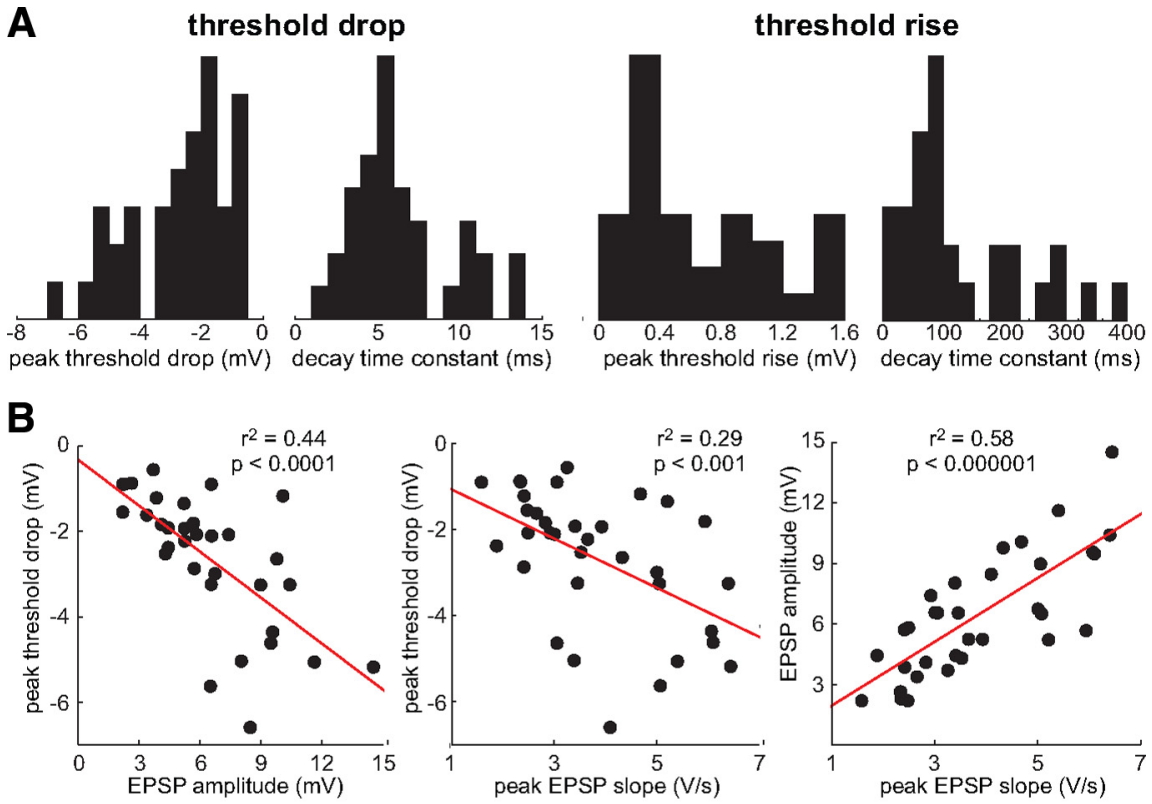

C
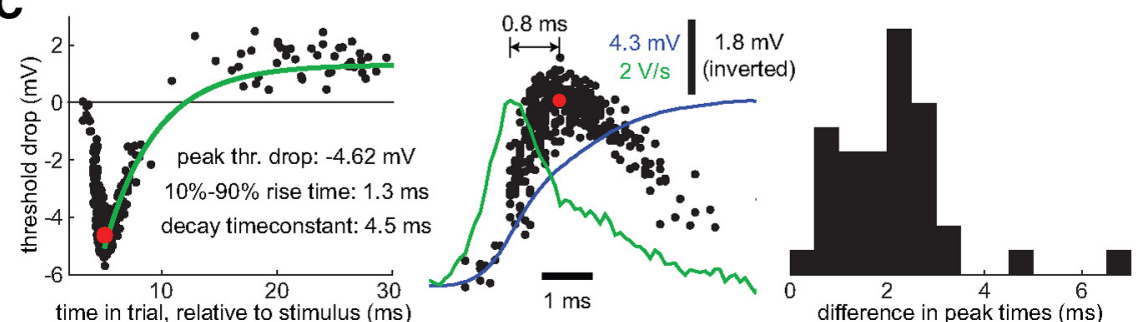

Figure 6. Distribution of threshold changes and their relationship to EPSPS. A, Left, Histogram of peak threshold drops $(0.5 \mathrm{mV}$ bins) and decay time constants ( $1 \mathrm{~ms}$ bins) from all cells ( $n=32$ ). Three outliers with decay time constants of 20.1, 30.2, and 47.7 ms are not shown. Right, Histogram of peak threshold rises $(0.2 \mathrm{mV}$ bins, $n=32$ ) and decay time constants ( $20 \mathrm{~ms}$ bins, $n=30$, excluding 2 cells with no detectable threshold rise). A drop in threshold invariably preceded any threshold rise. $B$, Peak threshold drop as a function of EPSP size, as measured by amplitude (left) or peak depolarization rate (middle), shown with linear regression $p$ value and coefficient of determination. Red lines show linear fits. Right, Relationship between EPSP amplitude and peak slope. $\boldsymbol{C}$, Timing relationship between EPSPs and threshold drops. Left, Example of EPSP-associated threshold drop, as in Figure 5B (different cell). Middle, Early phase of threshold drop plotted on an inverted scale with the EPSP slope (green) and voltage trajectory (blue), both derived from an average over trials that did not contain short-latency $(<20 \mathrm{~ms})$ spikes. Right, Histogram of time differences ( $0.5 \mathrm{~ms}$ bins) between EPSP peak slope and peak threshold drop for all cells ( $n=32$, like the $0.8 \mathrm{~ms}$ difference shown in the example).

combined synaptic and current pulse data and for each spike measured the average rate of depolarization from the onset of the stimulus (synaptic or current injection) to $0.6 \mathrm{~ms}$ before spike initiation (to avoid slope changes associated with the spike itself); the magnitude of depolarization was defined as the difference between the potential just before stimulus onset and the threshold for spontaneous spikes. The average threshold drop of spikes fired $<10 \mathrm{~ms}$ after the preceding spike was smaller than spikes fired at longer ISIs (data not shown); these spikes were excluded from additional analysis. To isolate the effect of depolarization rate on spike threshold, we considered only spikes preceded by large depolarization (15-20 mV; note that the magnitude of depolarization is simply determined by the level of hyperpolarization at the time the stimulus is delivered, not by the strength of the stimulus itself). We found that the threshold drop grows with depolarization rate up to $\sim 4 \mathrm{~V} / \mathrm{s}$ but is relatively insensitive to depolarization rate beyond that point (Fig. $7 \mathrm{C}$, left). To isolate the effect of depolarization magnitude, we considered only spikes preceded by rapid depolarization $(>4 \mathrm{~V} / \mathrm{s})$. We found a linear relationship between depolarization magnitude (equivalent to the level of hyperpolarization at stimulus onset) and threshold drop up to a depolarization of $\sim 15 \mathrm{mV}$ (Fig. $7 C$, right).

\section{Dynamic threshold can exert a profound effect on responses to stimulation and permits STN cells to act as coincidence detectors}

The static spike threshold assumed for our simple STN model is clearly incorrect, but it may not be obvious how a dynamic spike threshold could explain a bimodal distribution of latencies to the first spike after a stimulus. The key feature that generates this effect is the brevity of the threshold drop associated with rapid depolarization. EPSPs that are large enough to cross the lowered spike threshold trigger spikes much earlier than they would with a static spike threshold (Fig. $8 \mathrm{~A}$, left). However, EPSPs that fall just shy of that lowered threshold trigger spikes considerably later than slightly larger EPSPs, because the threshold is rising faster than the membrane potential, and ultimately they trigger spikes at or near the baseline (spontaneous) threshold (Fig. 8A, left). Over many trials, a transient EPSPtriggered drop in spike threshold will cause a subset of spikes to be fired earlier, time-locked to the EPSP, whereas the timing of other spikes is essentially unchanged. This creates a gap in the spike latency distribution that resembles the effect of inhibition. Larger threshold drops shift spike timing on a larger fraction of trials, widening the gap, whereas more slowly decaying threshold drops soften the distinction between spikes that are advanced and those that are unchanged, eroding the gap (Fig. $8 \mathrm{~A}$, right). In addition to explaining the apparent inhibition in the response of STN cells to cortical input, EPSP-triggered threshold drops almost certainly contribute to the short latency of the response to cortical stimulation observed in vivo.

The preceding analysis assumes that an EPSP is linked to a particular temporal sequence of empirically measured spike threshold changes without explaining how the size and shape of the EPSP affects the magnitude and time course of the change. To obtain a simple model that can predict spike threshold under arbitrary patterns of input, we treat spike threshold itself as a dynamical variable that relaxes to a voltage-dependent steadystate value with first-order kinetics. The steady-state spike threshold of the model is a linear function of membrane potential, based on our data from rapid depolarizations (Fig. 7C, right), and we let the threshold decay to its steady-state value with a time constant of $6 \mathrm{~ms}$, comparable with the median value for the decay time constant of EPSP-evoked threshold drops $(5.7 \mathrm{~ms}$ ) (Fig. 6A, left). When this model was tested with single EPSPs, the resulting threshold changes measured as a function of spike latency (Fig. 
$8 B$, left) closely resembled experimentally observed changes. Specifically, threshold drops developed with a $10-90 \%$ rise time of $1.6-2.9 \mathrm{~ms}$, peaked $1.7-3.3 \mathrm{~ms}$ after the peak EPSP slope, and decayed with a time constant of 6.1-7.1 ms, depending on mean EPSP amplitude. Like experimentally observed threshold changes, the threshold drop decayed to a slightly elevated threshold $(0.2-0.9 \mathrm{mV}$ above spontaneous threshold). We do not model the slow recovery from this threshold rise; the threshold is reset at the beginning of every ISI. Peak threshold drop increased linearly with EPSP strength (as measured by peak EPSP slope) up to a limit of $\sim 6 \mathrm{mV}$ below spontaneous threshold (Fig. $8 B$, right), slightly smaller than the maximal threshold drops observed experimentally with EPSPs $(-6.6 \mathrm{mV})$ or current pulses $(-6.9 \mathrm{mV})$.

This model encodes the arrival rate of EPSPs, accurately tracking relatively slow changes in input rate (Fig. $8 C$, left) and generating an output rate that is a linear function of the input rate (Fig. $8 C$, right). Its rate coding performance is essentially identical to a model with a static spike threshold (Fig. 8C). Conversely, the dynamic threshold of this model could make it fire preferentially to coincident synaptic input that produces rapid depolarization. Even a cell with a static threshold can detect a coincidence that produces a sufficiently large depolarization. An effective coincidence detector, however, should have enhanced sensitivity to coincident input and, if capable of responding to noncoincident input, should offer a means for its postsynaptic targets to distinguish coincidence-triggered spikes from other spikes. We tested the capacity of our model for coincidence detection by examining its response to 50 small EPSPs distributed in time according to a Gaussian function of variable width. As the width of the Gaussian falls below $10 \mathrm{~ms}$, the peak firing rate of the dynamic threshold model grows more rapidly than the static threshold model, becoming twice as large for perfectly synchronous input (Fig. $8 D$, left). Furthermore, the firing pattern of the dynamic threshold model changes (and deviates from the static threshold model) as the input becomes more coincident: spikes fired after coincident input are either time-locked to the stimulus with a short delay ( $\sim 5 \mathrm{~ms}$ ) or occur considerably later $(>40 \mathrm{~ms}$ after the stimulus) (Fig. $8 D$, right), leaving a gap in the response PSTH that resembles inhibition. The occurrence of coincident input to the STN could be detected and distinguished from more slowly integrated input by monitoring the activity of a small population of STN cells that share that input; their population activity would contain synchronized spikes encoding the timing of the input followed by brief silence.

0.5 ms current pulses $(+300 \mathrm{pA})$
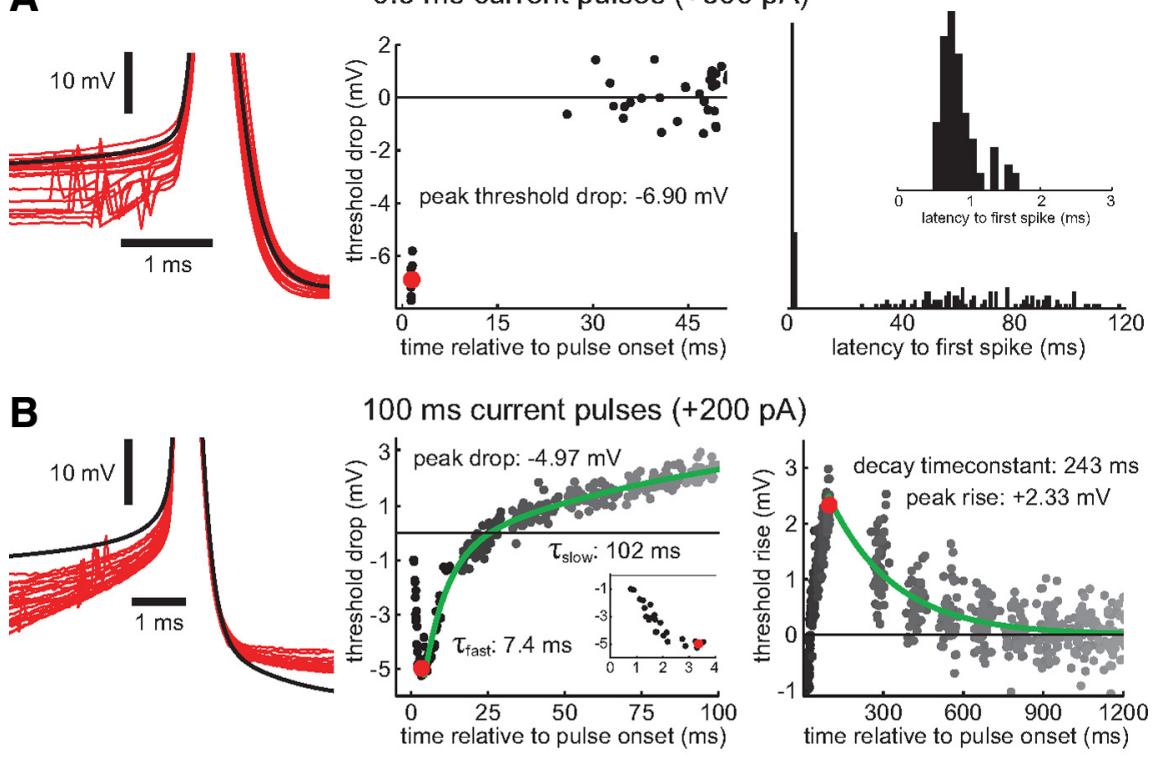

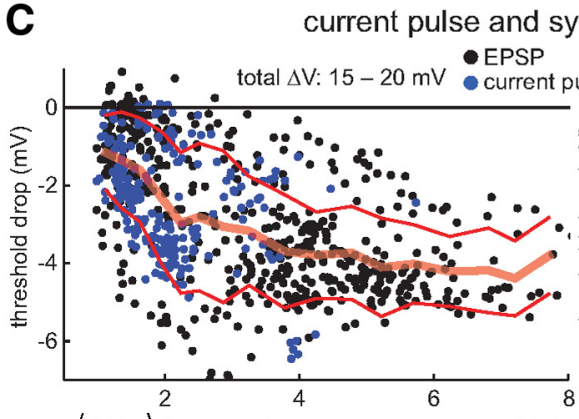

Figure 7. Somatic voltage trajectory predicts threshold change. $\boldsymbol{A}$, Example of threshold changes induced by very brief $(0.5 \mathrm{~ms})$ current pulses. Left, Examples of first spike after current pulse (red) and the average spontaneous spike (black). Middle, Relative threshold of individual spikes as a function of time since current pulse onset (black circles); red circle marks peak threshold drop. Right, Histogram of latency to first spike (measured from pulse onset), $1 \mathrm{~ms}$ bins. Inset shows detail of short-latency peak with 0.1 current pulse onset; red circle marks peak threshold drop, and the green line is a biexponential fit. Inset shows detail of early phase ( (black circles) and current injection (blue circles); each circle corresponds to a single poststimulus spike, mean \pm SD. Right, As above but plotting relative spike threshold as a function of prestimulus potential (relative to spontaneous spike threshold) for rapid depolarizations ( $>4 \mathrm{~V} / \mathrm{s}$ ). Bin widths for mean \pm SD (red lines) are 0.5 or $1 \mathrm{mV}$, and green line shows linear fit from data with more depolarized starting potentials (more than $-14 \mathrm{mV}$ ).

\section{Discussion}

Signaling in the basal ganglia is often assumed to be mediated by changes in firing rates, changes that might, for example, release basal ganglia targets from tonic inhibition to facilitate the initiation or selection of an action (Albin et al., 1989; Chevalier and Deniau, 1990). The use of rate coding implies good temporal integration of synaptic inputs, a feature that is usually limited by membrane conductances that allow synaptically delivered charge to leak out of the cell over time. We found that STN cells exhibit a balance between active and passive conductances that gives them an effectively zero membrane conductance-and hence an effectively infinite membrane time constant_-over a wide voltage range, consistent with the hypothesis that STN cells are good rate-coding integrators. We also found that STN cells exhibit 

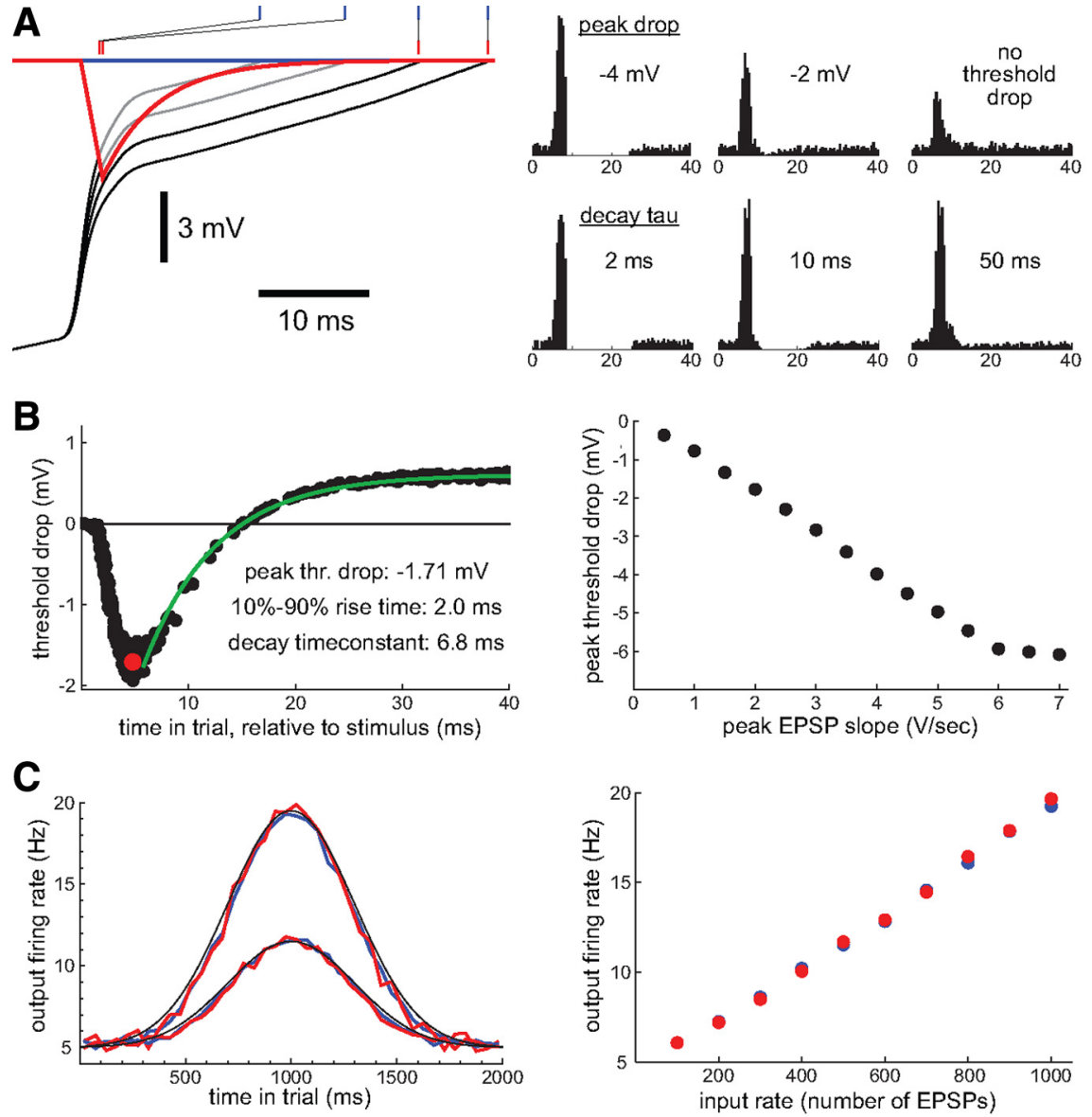

D

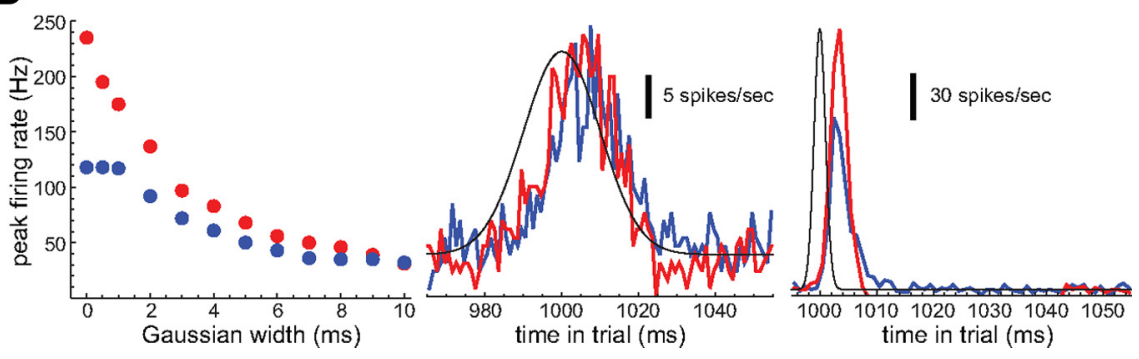

Figure 8. How dynamic threshold can shape the response of STN cells. $A$, Adding an EPSP-triggered threshold drop to the model shown in Figure 4D predicts a bimodal latency distribution. Left, Simulated EPSPs of varying amplitude with dynamic (red) and static (blue) threshold. Rasters (top) mark spike times in the presence (red) and absence (blue) of dynamic threshold. Right, Histograms of latency to first poststimulus spike as peak threshold drop (top row, with decay time constant of $5 \mathrm{~ms}$ ) and threshold drop decay time constant (bottom row, with peak threshold drop of $-4 \mathrm{mV}$ ) are varied. $\boldsymbol{B}$, Threshold behavior in a model that treats threshold as a dynamical variable with first-order kinetics and a voltage-dependent steady state. Left, Model threshold as a function of spike latency when stimulus is an EPSP with a peak slope of $2 \pm 0.1 \mathrm{~V} / \mathrm{s}$. Right, Peak threshold drop as a function of mean peak EPSP slope (slope SD is $5 \%$ of mean). C, Rate coding performance of the model. Left, Simulated PSTH (1000 trials, 50 ms bins), in which stimulus is a large number ( 500 and 1000 ) of small EPSPs $(0.1 \mathrm{~V} / \mathrm{s}$ peak slope) distributed randomly across the trial (EPSP onset time follows Gaussian distribution with mean of $1000 \mathrm{~ms}$ and SD of $300 \mathrm{~ms})$. Blue lines show response with static threshold, and red lines show response with dynamic threshold. Thin black lines show time course of average EPSP onsets (arbitrary vertical scale). Right, Mean output firing rate, averaged over a $100 \mathrm{~ms}$ window centered on the Gaussian peak ( $1000 \mathrm{~ms}$ trial time, same width as in (), as a function of input rate (measured by total number of $0.1 \mathrm{~V} / \mathrm{s}$ EPSPs distributed across each trial) under static (blue) and dynamic (red) spike threshold. D, Coincidence detection performance of the model. Left, Peak firing rate (from PSTHs with $1 \mathrm{~ms}$ bins) as a function of Gaussian width controlling the distribution of $50 \mathrm{small}(0.1 \mathrm{~V} / \mathrm{s})$ EPSPs delivered on each trial, with static (blue) and dynamic (red) threshold. Middle and Right, PSTHs (1000 trials, $1 \mathrm{~ms}$ bins) as in C, save that stimulus is 50 EPSPs distributed by Gaussians of $10 \mathrm{~ms}$ (middle) and $1 \mathrm{~ms}$ (right) widths. Note that thin black lines show distributions of EPSP onsets; peak synaptic drive lags by $\sim 2 \mathrm{~ms}$.

robust but transient decreases in spike threshold when depolarized rapidly. Such a dynamically changing threshold is of no evident use in accurately integrating synaptic inputs, but it does enhance the sensitivity of STN cells to coincident input. STN cells potentially combine two functions-integration and coincidence detection-that are normally considered incompatible.

Although STN cells can potentially act as good integrators, they may not function as perfectly linear and leak-free integrators in vivo. We have not shown that EPSPs sum linearly in STN cells, nor that their impact is independent of the phase of the ISI at which they arrive. Nevertheless, the integrative properties of STN cells in brain slices are not limited by their membrane time constant. STN cells may not retain a near-zero membrane slope conductance in vivo, in which the synaptic conductance load is presumably much greater. The persistent $\mathrm{Na}^{+}$current presumably cannot grow to continue balancing vastly greater ohmic conductances, but synaptic inputs themselves might recruit additional negative slope conductances, e.g., voltage-gated calcium channels (Song et al., 2000) or NMDA-type glutamate receptors. Even if the near-perfect balance between passive and active conductances is merely an accident occurring in brain slices, the persistent $\mathrm{Na}^{+}$ current will reduce the slope conductance and lengthen the membrane time constant, thereby improving integrative performance.

However effective STN cells may be at integration and rate coding, their dynamic spike threshold enhances their sensitivity to temporally coincident synaptic input. Coincidence detection is conventionally accomplished by using a short membrane time constant that permits otherwise quiescent cells to fire only during coincident input (König et al., 1996). STN cells, by contrast, can fire without any input whatsoever, and thus functional coincidence detection by STN cells requires a method to identify coincidenceencoding spikes. Our results show that the transient threshold drops induced by larger EPSPs not only facilitate the triggering of time-locked spikes, they change the firing pattern to create a sharp increase in spiking followed by a pause, a pattern that could be readily detected in the activity of a small population of STN cells sharing coincident input. Curiously, the timing of this intrinsically generated pause overlaps with the timing of polysynaptic inhibition arriving from the GPe in STN cells recorded in vivo, inhibition that is most likely triggered by the shortlatency burst occurring in the STN (Kita et al., 2004). Thus, STN cells are embedded in basal ganglia circuitry that reinforces the firing pattern associated with coincidence detection. Moreover, the STN and the GPe project to the same principal structures (Smith et al., 1998), so that the 
GPe can simultaneously sharpen the burst-pause response in the STN and directly reinforce that pattern in STN targets.

We propose a new mode of basal ganglia operation in which the STN and GPe jointly produce a brief, short-latency excitation-inhibition sequence that is triggered by rapid changes in cortical drive to the STN. This pattern is transmitted to the main output nuclei of the basal ganglia where it can rapidly affect basal ganglia targets in the thalamus and midbrain and is also transmitted to midbrain dopaminergic neurons (Smith et al., 1998) where it could control the generation of bursts associated with reward prediction or behavioral salience (Schultz, 2007; Redgrave et al., 2008). The role of the striatum in this mode may be primarily to modulate the generation of and response to the excitation-inhibition sequence by influencing the recruitment of GPe cells by the STN and by influencing the response of basal ganglia output nuclei to STN and GPe activity. This mode of operation could coexist with other modes, including a rate coding mode in which corticostriatal pathways can play a more prominent role. Although electrical stimulation of the cortex can produce this excitatory-inhibitory sequence and the dynamic threshold phenomenon we observed should facilitate its generation, several aspects of this hypothesis are as yet untested. For example, it is not clear how frequently naturally occurring cortical activity would briefly synchronize a functionally significant population of STN cells or whether such a population could in turn synchronize a population of GPe cells that innervates the activated STN population and/or the projection targets of that population. Nevertheless, the fact that the conduction velocities of subthalamopallidal and subthalamonigral axons are adjusted to produce the same average conduction time despite large differences in path length (Kita et al., 1983) suggests that timing of STN spikes is important under some circumstances, which would not be true if the STN uses a rate code exclusively.

The dynamic threshold we observed in STN cells is almost certainly a manifestation of the threshold accommodation phenomenon long known in a variety of preparations (e.g., Bradley and Somjen, 1961; Schlue et al., 1974; Wickens and Wilson, 1998). In cortical neurons, dynamic threshold changes have been reported to contribute to stimulus selectivity (Wilent and Contreras, 2005) and, as we suggest for STN cells, to promote coincidence detection (Azouz and Gray, 2000). Conversely, a recent study of spike initiation in cortical pyramidal neurons found that the spike threshold measured in the soma could vary widely even as the axonal spike threshold remained relatively constant (Yu et al., 2008). This occurs because the cable properties of the axon filter out rapid fluctuations in somatic voltage, and thus the potential in the soma and axon diverge most under the very conditions when the dynamic threshold mechanism is supposedly engaged. Yu et al. (2008) argue that much of threshold change reported during rapid somatic potential changes is an artifact of this filtering and that real threshold variation at the site of spike initiation, i.e., the axon, is limited; they therefore suggest that the contribution of dynamic threshold to neuronal function may be less than was supposed. Because spikes are axonally initiated in STN cells (Atherton et al., 2008), this criticism could apply to our results as well. However, somatodendritic current injection or conductance changes that cause rapid somatic depolarization should generate smaller and slower depolarization in the axon. If the axonal threshold remains constant, spikes would be triggered at a higher apparent somatic threshold under these conditions, whereas we observed lower thresholds. Although divergence between somatic and axonal potentials undoubtedly contributes to apparent threshold variability as Yu et al. (2008) argue, it should actually mute the effect we report here.

Recent work on dynamic threshold has emphasized the role of $d V / d t$ in setting the threshold. We found that the membrane potential before depolarization onset is also critical, which is expected if threshold changes are controlled by a voltage-dependent gating variable such as $\mathrm{Na}^{+}$channel inactivation. Rapid depolarization enables threshold drops, but the maximum size of the drop is determined by the degree of sustained hyperpolarization that precedes it; neglect of this factor may explain why Yu et al. (2008) observed low threshold variability. We also improve on past work by quantitatively characterizing the dynamics of threshold changes, which affect the firing patterns generated under this phenomenon. We were able to capture threshold dynamics with a simple model defined by just three parameters.

Understanding the influence synaptic inputs exert on the spiking output of a neuron can be fairly described as the central problem of cellular neurophysiology. The dynamical behavior of spike threshold is certainly an essential part of such an understanding for STN cells. For example, although EPSPs and IPSPs move the membrane potential in opposite directions, they can trigger spikes cooperatively through their effects on threshold; indeed, large IPSPs can produce a drop in spike threshold that long outlasts their effect on membrane potential (Baufreton et al., 2005). Dynamic threshold phenomena are likely to be widespread if not universal, but the voltage dependence and kinetics of dynamic threshold may vary widely, depending on the distribution of $\mathrm{Na}^{+}$channels, the geometry and cable properties of the spike initiation zone, and the voltage dependence and kinetics of $\mathrm{Na}^{+}$inactivation. These factors, like other cellular properties, may be tuned to support specific functions, and some of them may be subject to modulation. For most cell types, threshold dynamics will probably have to be probed experimentally to fully grasp their input-output relationship. Fortunately, a simple modeling approach can capture dynamic threshold properties with parameters that can be estimated from current-clamp data, allowing one to account for this functionally important property without using biophysically and morphologically detailed models defined by a vast array of unknown parameters.

\section{References}

Albin RL, Young AB, Penney JB (1989) The functional anatomy of basal ganglia disorders. Trends Neurosci 12:366-375.

Atherton JF, Wokosin DL, Ramanathan S, Bevan MD (2008) Autonomous initiation and propagation of action potentials in neurons of the subthalamic nucleus. J Physiol [Erratum (2009) 587:709] 586:5659-5700.

Azouz R, Gray CM (2000) Dynamic spike threshold reveals a mechanism for synaptic coincidence detection in cortical neurons in vivo. Proc Natl Acad Sci U S A 97:8110-8115.

Baufreton J, Atherton JF, Surmeier DJ, Bevan MD (2005) Enhancement of excitatory synaptic integration by GABAergic inhibition in the subthalamic nucleus. J Neurosci 25:8505-8517.

Bevan MD, Wilson CJ (1999) Mechanisms underlying spotaneous oscillation and rhythmic firing in rat subthalamic neurons. J Neurosci 19:7617-7628.

Bradley K, Somjen GG (1961) Accommodation in motoneurones of the rat and the cat. J Physiol 156:75-92.

Canteras NS, Shammah-Lagnado SJ, Silva BA, Ricardo JA (1990) Afferent connections of the subthalamic nucleus: a combined retrograde and anterograde horseradish peroxidase study in the rat. Brain Res 513:43-59.

Chevalier G, Deniau JM (1990) Disinhibition as a basic process in the expression of striatal functions. Trends Neurosci 13:277-280.

Do MT, Bean BP (2003) Subthreshold sodium currents and pacemaking of subthalamic neurons: modulation by slow inactivation. Neuron 39:109-120.

Fujimoto K, Kita H (1993) Response characteristics of subthalamic neurons 
to the stimulation of the sensorimotor cortex in the rat. Brain Res 609:185-192.

Hartigan JA, Hartigan PM (1985) The dip test of unimodality. Ann Stat 13:70-84.

Kempter R, Gerstner W, van Hemmen JL (1998) How the threshold of a neuron determines its capacity for coincidence detection. BioSystems 48:105-112.

Kita H (1994) Physiology of two disynaptic pathways from the sonsorimotor cortex to the basal ganglia output nuclei. In: Basal ganglia IV (Percheron G, ed), pp 263-276. New York: Plenum.

Kita H, Chang HT, Kitai ST (1983) Pallidal inputs to subthalamus: intracellular analysis. Brain Res 264:255-265.

Kita H, Nambu A, Kaneda K, Tachibana Y, Takada M (2004) Role of ionotropic glutamatergic and GABAergic inputs on the firing activity of neurons in the external pallidum in awake monkeys. J Neurophysiol 92: 3069-3084.

Kitai ST, Deniau JM (1981) Cortical inputs to the subthalamus: intracellular analysis. Brain Res 214:411-415.

Kitano H, Tanibuchi I, Jinnai K (1998) The distribution of neurons in the substantia nigra pars reticulata with input from the motor, premotor, and prefrontal areas of the cerebral cortex in monkeys. Brain Res 784:228238.

König P, Engel AK, Singer W (1996) Integrator or conincidence detector? The role of the cortical neuron revisited. Trends Neurosci 19:130-137.

Magill PJ, Sharott A, Bevan MD, Brown P, Bolam JP (2004) Synchronous unit activity and local field potentials evoked in the subthalamic nucleus by cortical stimulation. J Neurophysiol 92:700-714.

Nambu A, Tokuno H, Hamada I, Kita H, Imanishi M, Akazawa T, Ikeuchi Y,
Hasegawa N (2000) Excitatory cortical inputs to pallidal neurons via the subthalamic nucleus in the monkey. J Neurophysiol 84:289-300.

Redgrave P, Gurney K, Reynolds J (2008) What is reinforced by phasic dopamine signals? Brain Res Rev 58:322-339.

Ryan LJ, Clark KB (1991) The role of the subthalamic nucleus in the response of globus pallidus neurons to stimulation of the prelimbic and agranular frontal cortices in rats. Exp Brain Res 86:641-651.

Schlue WR, Richter DW, Mauritz KH, Nacimiento AC (1974) Responses of cat spinal motoneuron somata and axons to linearly rising currents. J Neurophysiol 37:303-309.

Schultz W (2007) Behavioral dopamine signals. Trends Neurosci 30:203-210.

Smith Y, Bevan MD, Shink E, Bolam JP (1998) Microcircuitry of the direct and indirect pathways of the basal ganglia. Neuroscience 86:353-387.

Song WJ, Baba Y, Otsuka T, Murakami F (2000) Characterization of $\mathrm{Ca}^{2+}$ channels in rat subthalamic nucleus neurons. J Neurophysiol 84:26302637.

Tachibana Y, Kita H, Chiken S, Takada M, Nambu A (2008) Motor cortical control of internal pallidal activity through glutamatergic and GABAergic inputs in awake monkeys. Eur J Neurosci 27:238-253.

Wickens JR, Wilson CJ (1998) Regulation of action-potential firing in spiny neurons of the rat neostriatum in vivo. J Neurophysiol 79:2358-2364.

Wilent WB, Contreras D (2005) Stimulus-dependent changes in spike threshold enhance feature selectivity in rat barrel cortex. J Neurosci 25:2983-2991.

Yu Y, Shu Y, McCormick DA (2008) Cortical action potential backpropagation explains spike threshold variability and rapid-onset kinetics. J Neurosci 28:7260-7272. 